Pacific Journal of Mathematics

LOCAL ISOMORPHISM OF COMPACT CONNECTED LIE 


\title{
LOCAL ISOMORPHISM OF COMPACT CONNECTED LIE GROUPS
}

\author{
P. F. BAUM
}

\begin{abstract}
It is shown that two nonisomorphic compact connected Lie groups can be covering groups of each other. This is examined in detail and is related to the question of determining all the compact connected Lie groups belonging to a fixed Lie algebra.
\end{abstract}

Given a Lie group $G$ it is natural to ask: "What are all the groups locally isomorphic to G?" The answer to this is well known. If $\bar{G}$ denotes the simply connected covering group of $G$, then any group locally isomorphic to $G$ is obtained by forming the quotient group $\bar{G} / \Gamma$ where $\Gamma$ is a discrete subgroup of the center of $\bar{G}$. (For this see e.g. [3]). We shall say that a Lie group $G_{1}$ covers a Lie group $G_{2}$ if there exists a continuous homomorphism of $G_{1}$ onto $G_{2}$ with discrete kernel. We can then pose the question: "Let $G$ be a Lie group and let $G_{1}, G_{2}, \cdots$ be Lie groups such that any group locally isomorphic to $G$ is isomorphic to one and only one of the $G_{i}$. Order the set $G_{1}, G_{2}, \cdots$ by setting $G_{i} \geqq G_{j}$ if $G_{i}$ covers $G_{j}$. How can one describe the structure of this ordered set?" In the case when $G$ is compact connected we give a precise answer to this question.

One surprising result of the investigation is that two compact connected Lie groups can cover each other but not be isomorphic. The simplest example of this is provided by the groups $U(5)$ and $U(5) / \Gamma_{2}$, where $U(5)$ denotes the $5 \times 5$ unitary group and $\Gamma_{2}$ is the subgroup of $U(5)$ consisting of the two matrices $\pm I, \quad I=$ identity matrix.

Closely related to this example is the:

Proposition. In $U(n)$ let $\Gamma_{k}$ denote the subgroup of all diagonal matrices $\lambda I$ where $\lambda$ is a complex number such that $\lambda^{k}=1$. Then $U(n) / \Gamma_{k_{1}}$ and $U(n) / \Gamma_{k_{2}}$ are isomorphic if and only if $k_{1} \equiv \pm k_{2} \bmod n$.

1. Notation. If $G$ is a compact connected Lie then $\widetilde{G}$ shall denote the covering group of $G$ of the form $H_{1} \times H_{2} \times \cdots \times H_{t} \times T^{n}$ where each $H_{i}$ is a compact connected simply connected group in one of the Cartan series $A_{n}, B_{n}, C_{n}, D_{n}$ or is a compact connected simply connected exceptional group, and $T^{n}$ is the $n$-dimensional torus group. $T^{n}=\left\{\left(z_{1}, z_{2}, \cdots, z_{n}\right) \mid z_{i} \in C\right.$ and $\left.\left|z_{i}\right|=1\right\}$.

If $K$ is a subgroup of the center of $H_{1} \times H_{2} \times \cdots \times H_{t}$ and $\varphi$ is a homomorphism of $K$ into $T^{n}$, then $(K, \varphi)$ denotes the subgroup of $\widetilde{G}$ consisting of all elements $(g, \varphi(g))$ where $g$ ranges over $K$. 
2. Proposition. If $G$ is a compact connected Lie group and $G_{1}$ is a compact connected Lie group locally isomorphic to $G$, then there exists a subgroup $K$ of the center of $H_{1} \times \cdots \times H_{t}$ and a homomorphism $\varphi: K \rightarrow T^{n}$ such that $G_{1}$ is isomorphic to $\widetilde{G} /(K, \varphi)$.

Proof. If suffices to show that any group $\widetilde{G} / \Gamma$, where $\Gamma$ is a discrete subgroup of the center of $\widetilde{G}$, is isomorphic to a group of the form $\widetilde{G} /(K, \varphi)$. So given $\Gamma$ let $p(\Gamma)=\left\{g \in T^{n} \mid(1, g) \in \Gamma\right\}$. Here 1 denotes the identity element of $H_{1} \times H_{2} \times \cdots \times H_{t}$. Then $T^{n} / p(\Gamma)$ is isomorphic to $T^{n}$ so there exists a homomorphism $f$ of $T^{n}$ onto itself with Kernel $f=p(\Gamma)$. Define a homomorphism $\widetilde{f}: \widetilde{G} \rightarrow \widetilde{G}$ by $\widetilde{f} \mid T^{n}=f$ and $\tilde{f} \mid H_{1} \times \cdots \times H_{t}=$ the identity map. Then $\tilde{f}$ is a homomorphism of $\widetilde{G}$ onto itself, $\widetilde{f}(\Gamma)$ is a group of the form $(K, \varphi)$ and $\widetilde{f}^{(-1)}(K, \varphi)=\Gamma$. So $\widetilde{f}$ gives an isomorphism of $\widetilde{G} / \Gamma$ onto $\widetilde{G} /(K, \varphi)$.

3. Corollary. If $G$ is a compact connected Lie group then to within isomorphism of Lie groups there exist only finitely many compact connected Lie groups locally isomorphic to $G$.

Proof. In $\widetilde{G}$ there are only finitely many subgroups of the form $(K, \varphi)$.

4. Definition. A subgroup of $\widetilde{G}$ of the form $(K, \varphi)$ shall be called a special subgroup of $\widetilde{G}$.

5. Proposition. Let $G$ be a compact connected Lie group and let $\left(K_{1}, \varphi_{1}\right)\left(K_{2}, \varphi_{2}\right)$ be two special subgroups of $\widetilde{G}$. Let $h: \widetilde{G} /\left(K_{1}, \varphi_{1}\right) \rightarrow$ $\widetilde{G} /\left(K_{2}, \varphi_{2}\right)$ be a covering homomorphism and let $\pi_{i}: \widetilde{G} \rightarrow \widetilde{G} /\left(K_{i}, \varphi_{i}\right)$ be the projection. Then there is a unique covering homomorphism $\widetilde{h}: \widetilde{G} \rightarrow \widetilde{G}$ such that the diagram

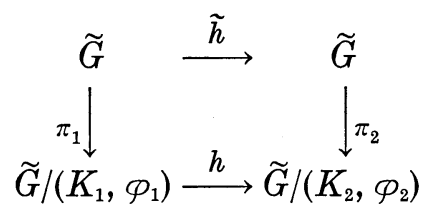

is commutative. If $h$ is an isomorphism then $\tilde{h}$ is an automorphism of $\widetilde{G}$.

Proof. $\pi_{i}$ maps $T^{n}$ isomorphically onto the identity component of the center of $\widetilde{G} /\left(K_{i}, \varphi_{i}\right)$. So $\widetilde{h} \mid T^{n}$ can be defined by $\widetilde{h} \mid T^{n}=\pi_{2}^{-1} \circ h \circ \pi_{1}$.

To define $\widetilde{h} \mid H_{1} \times \cdots \times H_{t}$ observe that $\pi_{2}^{-1} \circ h \circ \pi_{1}$ is well-defined in a neighborhood of the identity of $\widetilde{G}$. Thus $\pi_{2}^{-1} \circ h \circ \pi_{1}$ gives a local homomorphism of $H_{1} \times H_{2} \times \cdots \times H_{t}$ into $\widetilde{G}$ and since $H_{1} \times \cdots \times H_{t}$ 
is simply connected this extends to a homomorphism of $H_{1} \times \cdots \times H_{t}$ into $\widetilde{G}$.

Hence $\widetilde{h}$ is defined on all of $\widetilde{G}$. Since $\widetilde{G}$ is connected and locally one has $h \pi_{1}=\pi_{2} \tilde{h}$, one has this globally.

The uniqueness of $\widetilde{h}$ follows because $\widetilde{G}$ is connected and locally $\tilde{h}=\pi_{2}^{-1} \circ h \circ \pi_{1}$.

If $h$ is an isomorphism let $\eta=h^{-1}$. Then $\tilde{h} \tilde{\eta}$ and $\tilde{\eta} \tilde{h}$ are locally the identity mapping so this holds globally and $\tilde{\eta}=(\widetilde{h})^{-1}$.

REMARK. If $\Gamma_{1}$ and $\Gamma_{2}$ are arbitrary discrete central subgroups of $\widetilde{G}$ and $h: \widetilde{G} / \Gamma_{1} \rightarrow \widetilde{G} / \Gamma_{2}$ is a covering homomorphism then it is not in general true that $h$ can be lifted to a homomorphism $\widetilde{h}: \widetilde{G} \rightarrow \widetilde{G}$.

6. Corollary. $\widetilde{G} /\left(K_{1}, \varphi_{1}\right)$ covers (is isomorphic to) $\widetilde{G} /\left(K_{2}, \varphi_{2}\right)$ if and only if there exists an automorphism $\alpha$ of $H_{1} \times \cdots \times H_{t}$ and $a$ covering homomorphism $\beta$ of $T^{n}$ onto itself (and an automorphism $\beta$ of $T^{n}$ ) such that $\alpha$ maps $K_{1}$ into (onto) $K_{2}$ and the diagram

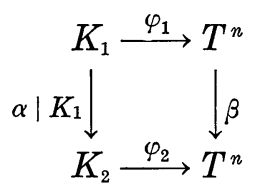

is commutative.

Proof. By applying the previous proposition and the fact that any homomorphism of $\widetilde{G}$ onto itself is of the form $\alpha \times \beta$ where $\alpha$ is an automorphism of $H_{1} \times \cdots \times H_{t}$ and $\beta$ is a covering homomorphism of $T^{n}$ onto itself.

7. Definition. A quasi-ordered set is a set $S$ together with a transitive relation $\geqq$, such that for all $x \in S, x \geqq x$. Two quasi ordered sets $S_{1}$ and $S_{2}$ are isomorphic if there exists a one-to-one and onto mapping of sets $f: S_{1} \rightarrow S_{2}$ such that for all $x, y \in S_{1}, x \geqq y$ if and only if $f(x) \geqq f(y)$.

8. Definition. Let $G$ be a compact connected Lie group and let $G_{1}, G_{2}, \cdots, G_{l}$ be compact connected Lie groups such that any compact connected Lie group locally isomorphic to $G$ is isomorphic to one and only one of the $G_{i}$. Define a transitive relation on the $G_{i}$ by setting $G_{i} \geqq G_{j}$ if $G_{i}$ covers $G_{j}$. The set $G_{1}, G_{2}, \cdots, G_{l}$ together with the relation $\geqq$ is called the local isomorphism system of $G$.

9. Definition. Let $G$ be a compact connected Lie group and let $\left(K_{1}, \varphi_{1}\right),\left(K_{2}, \varphi_{2}\right), \cdots,\left(K_{r}, \varphi_{r}\right)$ be the special subgroups of $\widetilde{G}$. Define an 
equivalence relation $\sim$ on the $\left(K_{i}, \varphi_{i}\right)$ by setting $\left(K_{i}, \varphi_{i}\right) \sim\left(K_{j}, \varphi_{j}\right)$ if there exist automorphisms $\alpha, \beta$ of $H_{1} \times \cdots \times H_{t}$ and $T^{n}$ such that $\alpha$ maps $K_{1}$ onto $K_{2}$ and the diagram

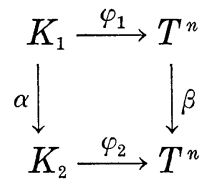

is commutative. Denote the equivalence class of $\left(K_{i}, \varphi_{i}\right)$ by $\left[\left(K_{i}, \varphi_{i}\right)\right]$. On the set of equivalence classes define a transitive relation $\geqq$ by $\left[\left(K_{i}, \varphi_{i}\right)\right] \geqq\left[\left(K_{j}, \varphi_{j}\right)\right]$ if there exists an automorphism $\alpha$ of $H_{1} \times \cdots \times H_{t}$ and a covering homomorphism $\beta$ of $T^{n}$ onto itself such that $\alpha$ maps $K_{1}$ into $K_{2}$ and the diagram

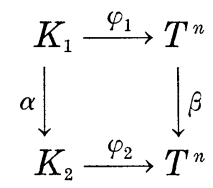

is commutative. The set of equivalence class $\left[\left(K_{i}, \varphi_{i}\right)\right]$ together with this transitive relation is the special subgroup system of $\widetilde{G}$.

10. Theorem. Let $G$ be a compact connected Lie group. Then the local isomorphism system of $G$ and the special subgroup system of $\widetilde{G}$ are isomorphic quasi-ordered sets.

Proof. Let $\mathscr{L}=\left\{G_{1}, G_{2}, \cdots, G_{l}\right\}$ be the local isomorphism system of $G$ and let $\mathscr{S}$ be the special subgroup system of $\widetilde{G}$. Map $\mathscr{S}$ to $\mathscr{L}$ by $[(K, \varphi)] \rightarrow$ that $G_{i}$ which is isomorphic to $\widetilde{G} /(K, \varphi)$. By Proposition 2 this map is onto and by Corollary 6 it is one-to-one.

REMARKs. The above theorem can be used to make actual computations. In doing a computation the inner automorphisms of $H_{1} \times H_{2} \times \cdots \times H_{t}$ are irrelevant since these have no effect on the $(K, \phi)$ because $K$ is a central subgroup of $H_{1} \times \cdots \times H_{t}$. Thus only the group Aut $\left(H_{1} \times \cdots \times H_{t}\right) /$ Inner Aut $\left(H_{1} \times \cdots \times H_{t}\right)$ plays a rôle. (Here Aut ( ) denotes the group of automorphisms of ( )). The group Aut $\left(H_{1} \times \cdots \times H_{t}\right) /$ Inner Aut $\left(H_{1} \times \cdots \times H_{t}\right)$ is a finite group whose structure may be read off the Dynkin diagram of $H_{1} \times \cdots \times H_{t}$ : Aut $\left(H_{1} \times \cdots \times H_{t}\right) /$ Inner Aut $\left(H_{1} \times \cdots \times H_{t}\right)$ is isomorphic to the group of symmetries of the Dynkin diagram. For this see [4].

Aut $\left(T^{n}\right)$ is isomorphic to the group of all $n \times n$ matrices with integer entries and determinant $= \pm 1$. The semi-group of all covering homomorphisms of $T^{n}$ onto itself is isomorphic to the semi-group of 
all $n \times n$ matrices with integer entries and nonzero determinant.

If $S$ is a quasi-ordered set then the structure of $S$ is conveniently represented by a diagram as follows:

Define an equivalence relation on $S$ by setting $x \sim y$ if $x \geqq y$ and $y \geqq x$. The set of equivalence classes forms a partially ordered set which may be diagrammed in the usual fashion (e.g. see [2]). To diagram $S$ diagram the associated partially ordered set and indicate the number of elements of $S$ in each equivalence class. For example

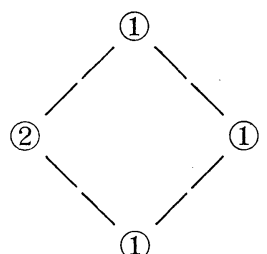

(1)

is the diagram of a quasi-ordered set with five elements $x_{1}, x_{2}, x_{3}, x_{4}, x_{5}$ and $\geqq$ defined by $x_{1} \geqq x_{i}, x_{i} \geqq x_{i}, x_{i} \geqq x_{5}$ for all $i$, and $x_{2} \geqq x_{3}, x_{3} \geqq x_{2}$.

EXAmples. If $G=U(n)$, then $\widetilde{G}=S U(n) \times S^{1}$ with a covering homomorphism $S U(n) \times S^{1} \rightarrow U(n)$ given by $(a, \lambda) \rightarrow a \lambda$. The center of $S U(n)$ is all diagonal matrices of the form $\mu I$ where $\mu^{n}=1$. This is a cyclic group of order $n$. $S U(n)$ has one outer automorphism and this is given by $\left(a_{i j}\right) \rightarrow\left(\overline{a_{i j}}\right)$. $\overline{a_{i j}}$ denotes the complex conjugate of $a_{i j}$. $S^{1}$ has one automorphism given by $\lambda \rightarrow \bar{\lambda}$. Let $\Gamma_{k}$ denote the subgroup of $U(n)$ consisting of all diagonal matrices $\theta I$ where $\theta^{k}=1$. Let $\omega=\cos 2 \pi / n+i \sin 2 \pi / n$. The inverse image of $\Gamma_{k}$ under the covering homomorphism of $S U(n) \times S^{1}$ onto $U(n)$ is all pairs of the form $\left(\omega^{i} I, \theta \omega^{-i}\right)$. Mapping $S U(n) \times S^{1}$ onto itself by $(a, \lambda) \rightarrow\left(\alpha, \lambda^{-k}\right)$ the pair $\left(\omega^{i} I, \theta \omega^{-i}\right)$ goes to $\left(\omega^{i} I, \omega^{k i}\right)$. Let $\Gamma$ denote the center of $S U(n)$ then $U(n) / \Gamma_{k}$ is isomorphic to $S U(n) \times S^{1} /\left(\Gamma, \varphi_{k}\right)$ where $\varphi_{k}$ is the homomorphism of $\Gamma$ into $S^{1}$ given by $\varphi_{k}(\omega I)=\omega^{k}$. From this and the above remark about the automorphisms of $S U(n) \times S^{1}$ it now follows that $U(n) / \Gamma_{k_{1}}$ and $U(n) / \Gamma_{k_{2}}$ are isomorphic if and only if $k_{1} \equiv \pm k_{2} \bmod n$. Thus $U(n)$ covers precisely $[n / 2]+1$ distinct Lie groups. $\quad([n / 2]=$ integer part of $n / 2)$.

If $n=5$ let $\Gamma=$ center of $S U(5)$ and let $\omega=\cos 2 \pi / 5+i \sin 2 \pi / 5$. There are 5 distinct homomorphisms $\varphi_{0}, \varphi_{1}, \varphi_{2}, \varphi_{3}, \varphi_{4}$ of $\Gamma$ into $S^{1}$ with $\varphi_{i}$ determined by $\varphi_{i}(\omega I)=\omega^{i}$. Thus in $S U(5) \times S^{1}$ there are six special subgroups: the special subgroup consisting of just the identity element and the $\left(\Gamma, \varphi_{i}\right)$. The automorphism of $S U(5) \times S^{1}$ given by $(\alpha, \lambda) \rightarrow(\alpha, \bar{\lambda})$ takes $\left(\Gamma, \varphi_{1}\right)$ onto $\left(\Gamma, \varphi_{4}\right)$ and takes $\left(\Gamma, \varphi_{2}\right)$ onto $\left(I, \varphi_{3}\right)$. Hence the special subgroup system of $S U(5) \times S^{1}$ has four distinct elements: $[(1,1)],\left[\left(\Gamma, \varphi_{0}\right)\right],\left[\left(\Gamma, \varphi_{1}\right)\right],\left[\left(\Gamma, \varphi_{2}\right)\right] .\left[\left(\Gamma, \varphi_{1}\right)\right] \geqq\left[\left(\Gamma, \varphi_{2}\right)\right]$ because $(\alpha, \lambda) \rightarrow\left(a, \lambda^{2}\right)$ takes $\left(\Gamma, \varphi_{1}\right)$ into $\left(\Gamma, \varphi_{2}\right)$. Also $\left[\left(\Gamma, \varphi_{2}\right)\right] \geqq\left[\left(\Gamma, \varphi_{1}\right)\right]$ because 
$(a, \lambda) \rightarrow\left(a, \lambda^{2}\right)$ takes $\left(\Gamma, \varphi_{2}\right)$ into $\left(\Gamma, \varphi_{1}\right)$. So the diagram of the local isomorphism system of $U(5)$ is:

At the top is the group $S U(5) \times S^{1}$. At the bottom is the group $S U(5) / \Gamma \times S^{1}$. In the middle are the two groups $U(5), U(5) /\{ \pm I\}$. Other computations can be made similarly.

The local isomorphism system of $U(15)$ is:

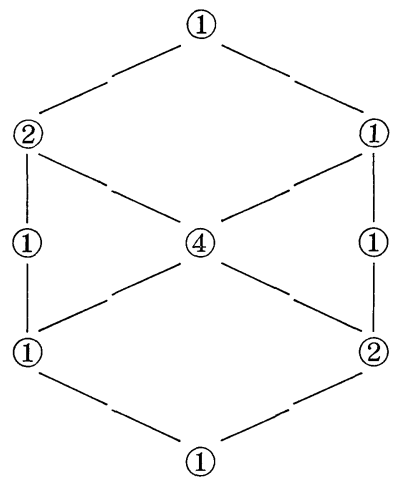

The local isomorphism system of $U(27)$ is:

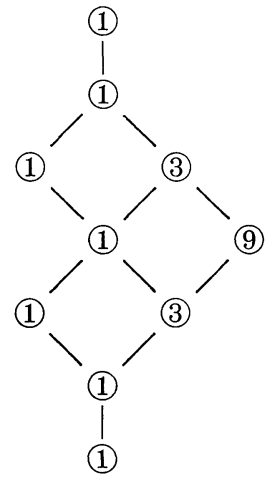


The local isomorphism system of $U(30)$ is:

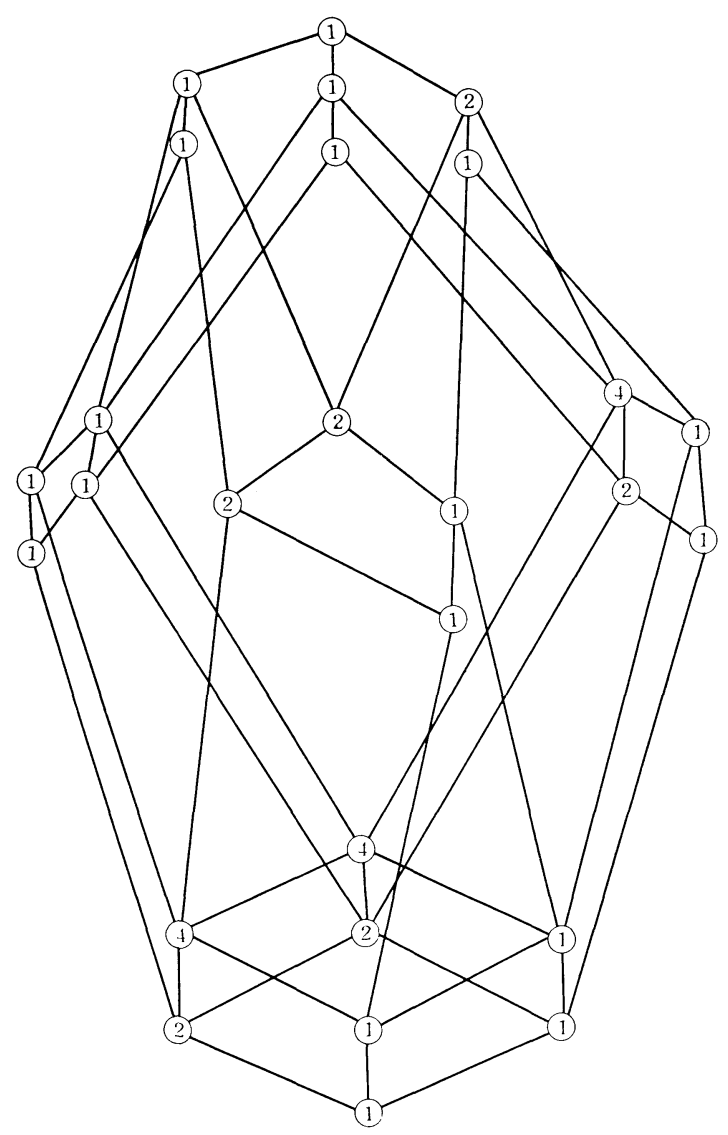

If two compact connected Lie groups cover each other, then as differentiable manifolds they are diffeomorphic. This holds because any compact connected Lie group is diffeomorphic to the Cartesian product of its maximal connected semi-simple subgroup and the identity component of its center. (This can be proved by applying the fact that a differentiable principal fibre bundle over a circle with connected structural group is trivial.)

Connected semi-simple Lie groups can be diffeomorphic as differentiable manifolds but not isomorphic as Lie groups. Examples of this are given in [1]. For compact connected simple (i.e. the only normal subgroups are discrete) Lie groups homotopy equivalence of the underlying differentiable manifolds implies isomorphism [1].

\section{REFERENCES}

1. P. F. Baum and W. Browder, The cohomology of quotients of classical groups, Topology 3 (1965), 305-336. 
2. G. Birkhoff, Lattice Theory, Amer. Math. Soc. Colloquium Publications 25 (1940).

3. C. Chevalley, Theory of Lie Groups, Princeton University Press, 1946.

4. N. Jacobson, Lie Algebras, Interscience Publishers, 1962.

Received August 1, 1966. This research was supported in part by National Science Foundation Grant NSF-GP-2425, Manifolds and Homological Algebra at Princeton University.

PRINCETON UNIVERSITY 


\section{PACIFIC JOURNAL OF MATHEMATICS}

\section{EDITORS}

\section{H. SAMELSON}

Stanford University

Stanford, California

J. P. JANS

University of Washington

Seattle, Washington 98105

\section{J. DugundJI}

University of Southern California Los Angeles, California 90007

RICHARD ARENS

University of California

Los Angeles, California 90024

\section{ASSOCIATE EDITORS}

E. F. BECKENBACH
B. H. NeUmanN

\section{SUPPORTING INSTITUTIONS}

UNIVERSITY OF BRITISH COLUMBIA CALIFORNIA INSTITUTE OF TECHNOLOGY

UNIVERSITY OF CALIFORNIA

MONTANA STATE UNIVERSITY

UNIVERSITY OF NEVADA

NEW MEXICO STATE UNIVERSITY

OREGON STATE UNIVERSITY

UNIVERSITY OF OREGON

OSAKA UNIVERSITY

UNIVERSITY OF SOUTHERN CALIFORNIA

\author{
STANFORD UNIVERSITY \\ UNIVERSITY OF TOKYO \\ UNIVERSITY OF UTAH \\ WASHINGTON STATE UNIVERSITY \\ UNIVERSITY OF WASHINGTON \\ AMERICAN MATHEMATICAL SOCIETY \\ CHEVRON RESEARCH CORPORATION \\ TRW SYSTEMS \\ NAVAL ORDNANCE TEST STATION
}

Mathematical papers intended for publication in the Pacific Journal of Mathematics should be typewritten (double spaced). The first paragraph or two must be capable of being used separately as a synopsis of the entire paper. It should not contain references to the bibliography. Manuscripts may be sent to any one of the four editors. All other communications to the editors should be addressed to the managing editor, Richard Arens at the University of California, Los Angeles, California 90024.

50 reprints per author of each article are furnished free of charge; additional copies may be obtained at cost in multiples of 50 .

The Pacific Journal of Mathematics is published monthly. Effective with Volume 16 the price per volume ( 3 numbers) is $\$ 8.00$; single issues, $\$ 3.00$. Special price for current issues to individual faculty members of supporting institutions and to individual members of the American Mathematical Society: $\$ 4.00$ per volume; single issues $\$ 1.50$. Back numbers are available.

Subscriptions, orders for back numbers, and changes of address should be sent to Pacific Journal of Mathematics, 103 Highland Boulevard, Berkeley 8, California.

Printed at Kokusai Bunken Insatsusha (International Academic Printing Co., Ltd.), 7-17, Fujimi 2-chome, Chiyoda-ku, Tokyo, Japan.

\section{PUBLISHED BY PACIFIC JOURNAL OF MATHEMATICS, A NON-PROFIT CORPORATION}

The Supporting Institutions listed above contribute to the cost of publication of this Journal, but they are not owners or publishers and have no responsibility for its content or policies. 


\section{Pacific Journal of Mathematics}

\section{Vol. 22, No. $2 \quad$ February, 1967}

Paul Frank Baum, Local isomorphism of compact connected Lie groups ....

Lowell Wayne Beineke, Frank Harary and Michael David Plummer, On the

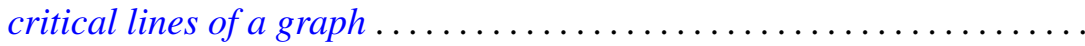

Larry Eugene Bobisud, On the behavior of the solution of the telegraphist's equation for large velocities .......................... 213

Richard Thomas Bumby, Irreducible integers in Galois extensions . . . . . . 221

Chong-Yun Chao, A nonimbedding theorem of nilpotent Lie algebras ..... 231

Peter Crawley, Abelian p-groups determined by their Ulm sequences ...... 235

Bernard Russel Gelbaum, Tensor products of group algebras ........... 241

Newton Seymour Hawley, Weierstrass points of plane domains .......... 251

Paul Daniel Hill, On quasi-isomorphic invariants of primary groups . . . . . 257

Melvyn Klein, Estimates for the transfinite diameter with applications to confomral mapping ................................ 267

Frederick M. Lister, Simplifying intersections of disks in Bing's side approximation theorem ............................. 281

Charles Wisson McArthur, On a theorem of Orlicz and Pettis ........... 297

Harry Wright McLaughlin and Frederic Thomas Metcalf, An inequality for

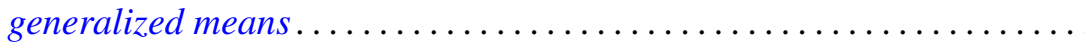

Daniel Russell McMillan, Jr., Some topological properties of piercing

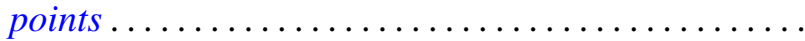

Peter Don Morris and Daniel Eliot Wulbert, Functional representation of topological algebras .

Roger Wolcott Richardson, Jr., On the rigidity of semi-direct products of Lie algebras..................................

Jack Segal and Edward Sandusky Thomas, Jr., Isomorphic

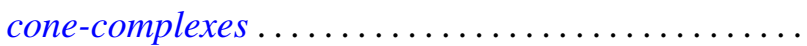

Richard R. Tucker, The $\delta^{2}$-process and related topics.... 